\title{
Nitric oxide production in critically ill patients
}

\author{
Hector R Wong, Joseph A Carcillo, Gilbert Burckart, Sandra S Kaplan
}

\begin{abstract}
Objective-To measure serum nitrite and nitrate levels in critically ill children as indicators of endogenous nitric oxide (NO) production.

Hypothesis-Endogenous NO production is increased in children with conditions characterised by immune stimulation.

Design-Prospective descriptive study in a multidisciplinary paediatric intensive care unit.
\end{abstract}

Patients-137 consecutive critically ill children with a variety of clinical conditions.

Interventions-Using a rapid microtitre plate technique, daily serum nitrite and nitrate levels were measured from serum samples that remained in the clinical laboratory after daily routine phlebotomy. Clinical and laboratory information was also gathered daily for each patient.

Results-The maximum serum nitrite plus nitrate levels $(\mu M)$ reached by children with infection (41.8 (SD 18.1)), sepsis syndrome (85.1 (39.9)), shock without sepsis (36.4 (19.1)), transplantation alone (61.0 (43.4)), transplantation with sepsis (200.7 (150.5)), or rejection (161.7 (70.4)), were higher than in controls (18.1 (9.3)). In the absence of exogenous NO donors, levels greater than $80 \mu M$ were reached only in children with the sepsis syndrome, organ transplantation, or acute rejection. Conclusions-Increased endogenous NO production occurs in children with clinical conditions associated with immune stimulation. Further investigation is warranted to determine the value of this simple and rapid test as a clinically useful diagnostic tool and therapeutic monitor in the evaluation of children at risk for the sepsis syndrome or acute allograft rejection.

(Arch Dis Child 1996;74:482-489)

Keywords: nitric oxide, sepsis, transplantation.

Correspondence to: Dr Hector R Wong, Division of Critical Care Medicine, Children's Hospital Medical Center, 3333 Burnet Avenue, Cincinnati, Ohio

45229-3039, USA.

Accepted 18 March 1996
In 1983 Wagner et al reported increased urinary nitrates in rats injected with endotoxin. ${ }^{1}$ During the next decade endogenously produced nitric oxide (NO) was identified as the source of this increase in nitrate and as an important effector molecule in proinflammatory conditions such as sepsis. ${ }^{2}$ Serum nitrite and nitrate, the stable oxidative end products of NO, have been reported in multiple animal and human studies as an indirect measure of in vivo whole body NO production. ${ }^{345-7} \mathrm{We}^{3}$ and other investigators ${ }^{45}$ have reported increased endogenous NO production in patients with the sepsis syndrome. These clinical studies are consistent with laboratory studies showing increased NO production in various mammalian cell types after stimulation with proinflammatory mediators. ${ }^{8} 9$ Immune stimulation, characterised by the elaboration of proinflammatory mediators, also occurs after solid organ transplantation, and during allograft rejection and infectious processes. Accordingly, increased NO production manifest as increased serum nitrite and nitrate levels is also likely to occur in these clinical conditions. ${ }^{10}$ Increased serum nitrite and nitrate levels may also be expected in clinical states which are not classically associated with immune activation. For example patients experiencing hypotension during haemodialysis ${ }^{11}$ or chronic heart disease ${ }^{12}$ have increased endogenous NO production. Administration of exogenous NO (either as inhaled NO or as intravenous NO donors) and decreased metabolism or elimination of serum nitrite and nitrate (at either the renal or hepatic level) are other potential causes of increased serum levels of these metabolites.

The development of a rapid microtitre plate technique to measure serum nitrite and nitrate levels allows for the potential use of these measurements as a clinical tool to evaluate endogenous NO production. Serum nitrites and nitrates are stable end products of NO production, not immediate indicators of NO production. In vitro studies show that nitrite and nitrate levels may not be detectable until 24 hours after immune stimulation and NO production. $^{89}$ Accordingly, a clinical state associated with increased NO production may not show increased nitrite and nitrate levels if evaluated too early in its evolution. Therefore, questions regarding increased NO production in specific clinical states may be more accurately evaluated by measuring the maximum nitrite and nitrate level attained during the course of an illness. 
1. Clinical suspicion of infection

2. Hyperthermia (rectal temperature $>38^{\circ} \mathrm{C}$ ) or hypothermia (rectal temperature $<35.0^{\circ} \mathrm{C}$ )

3. Tachycardia (heart rate $>2$ SD above the appropriate mean for age)

and

4. At least one of the following manifestations of inadequate or altered organ function and perfusion:

- Altered mental status (appropriate for age)

- Hypoxaemia $\left(\mathrm{PaO}_{2}<72\right.$ torr $\left.(9.5 \mathrm{kPa})\right)$ breathing room air, overt pulmonary disease not the direct cause of hypoxaemia

- Increased plasma lactate level

- Oliguria (urine output $<0.5 \mathrm{ml} / \mathrm{kg} / \mathrm{h}$ for at least one hour not due to decreased preload)

- Delayed capillary refill ( $>5$ seconds)

- Bounding peripheral pulses

Although increased serum nitrite and nitrate levels have been reported in two studies of neonates and children with the sepsis syndrome, ${ }^{57}$ no such information exists regarding the general and critically ill paediatric populations. The purposes of this prospective survey were: (1) to establish a range of values of serum nitrite and nitrate in critically ill children in whom increased NO is not expected (that is, in children without infection, sepsis, transplantation, rejection, or shock, and not being treated with exogenous NO donors); (2) to establish that serum nitrite and nitrate levels are a reasonable representation of NO by showing that nitrite and nitrate levels correlate with the concentration of exogenous NO donors received; (3) to establish that the maximum level of serum nitrite and nitrate reached by children with diseases thought to be associated with increased endogenous NO production (that is, infection, sepsis, shock without sepsis, transplantation, or rejection) are higher than the maximum level reached by critically ill children in whom endogenous NO production is most probably not increased; and (4) to examine the possibility that the use of threshold serum nitrite and nitrate levels may have clinical value as indicators or therapeutic monitors either at the time of diagnosis or during the course of illness in critically ill children with the sepsis syndrome or allograft rejection.

\section{Methods}

PATIENTS, DESIGN, AND DATA GATHERING Since no blood was drawn solely for study purposes, the human rights committee of Children's Hospital of Pittsburgh waived the need for parental consent. The intensive care unit at the Children's Hospital of Pittsburgh is a 23 bed tertiary multidisciplinary care facility that admits a complex population of surgical and medical patients. All children admitted to the intensive care unit were studied prospectively over a predetermined two month period. Patients were prospectively assigned daily, by an investigator unaware of serum concentrations of nitrite and nitrate, to one of the following defined groups: (1) exogenous NO: patients receiving exogenous sources of $\mathrm{NO}$ either as inhaled NO or as intravenous NO donors (sodium nitroprusside or glyceryl trinitrate); (2) infection: patients with a culture proven focus of infection (that is, bacteraemia, urinary tract infection, pneumonia) but not meeting criteria for the sepsis syndrome; (3) sepsis syndrome: see table 1 for definition based on a modification of the criteria of Bone et $a^{13}$ previously published by $\mathrm{us}^{7}$; (4) shock without sepsis: hypotension not associated with sepsis; (5) transplantation: patients who have undergone solid organ transplantation; (6) transplantation with infection; (7) transplantation with sepsis; (8) rejection: patients with acute allograft rejection defined as the requirement for aggressive antirejection therapy (antithymocyte gammaglobulin or OK-T3) added to the existing immunosuppressive regimen; or (9) controls: all critically ill children not meeting criteria for any one of the above clinical conditions.

One investigator, unaware of serum nitrite and nitrate concentrations, recorded data prospectively on each patient during each day of the study period. This investigator examined patients, reviewed case records, and recorded the age and diagnoses. The same investigator classified patients into one of the above mentioned groups. A second investigator, also unaware of serum nitrite and nitrate concentrations, recorded serum creatinine and bilirubin concentrations when available from daily laboratory test results. A third investigator collected serum from blood samples that remained in the clinical laboratory after routine daily phlebotomy and stored the samples at $4^{\circ} \mathrm{C}$. This investigator was unaware of all patient information except for patient name and medical record number. Decisions regarding the need for and frequency of phlebotomy were made by clinicians not involved in the study. We have not observed significant changes in serum nitrite and nitrate levels during storage (unpublished data).

\section{SERUM NITRITE AND NITRATE ASSAY}

Serum nitrite levels were measured using the Griess reaction. ${ }^{14}$ Serum samples were deproteinised using zinc sulphate at $56^{\circ} \mathrm{C}$ and the deproteinised samples were diluted $1: 1$ in ammonium chloride borate buffer, $\mathrm{pH}$ 8.5. Samples were then pipetted into 96 well microtitre dishes, and an equal volume of Griess reagent ( $1 \%$ sulphanilamide, $0.1 \%$ naphthyethylenediamine dihydrochloride, $2.5 \%$ orthophosphoric acid) was added to each well. After incubation for 15 minutes at room temperature, absorbance was measured at $550 \mathrm{~nm}$ using sodium nitrite as a standard.

Nitrate was evaluated in a similar manner, after first reducing serum nitrate to nitrite by placing the serum samples into the wells of 96 well Millipore filter plates treated with a slurry of freshly prepared deoxidised, washed cadmium powder in water. Samples were incubated for two hours at room temperature and then filtered into a plain 96 well plate using a 
Table 2 Serum nitrite plus nitrate levels at the time of diagnosis and maximum level attained during the course of illness

\begin{tabular}{|c|c|c|c|}
\hline & No & $\begin{array}{l}\text { Serum nitrite plus nitrate } \\
\text { levels at diagnosis mean } \\
\mu M(S D) \text { [median] }\end{array}$ & $\begin{array}{l}\text { Maximum serum nitrite } \\
\text { plus nitrate levels mean } \\
\mu M(S D) \text { [median] }\end{array}$ \\
\hline Controls & 56 & $17.4(9.3)[18]$ & $18.1(9.3)[18]$ \\
\hline Infection & 25 & $29.4(14.4)[27]$ & $41.8(18.1)[42] \dagger$ \\
\hline Sepsis & 12 & $75.1(39.9)[78]^{\star} \widehat{S}$ & $85.1(39.9)[78.5]+\pi$ \\
\hline Shock without sepsis & 8 & $28.2(13.9)[26.5]$ & $36.4(19.1)[36] \dagger$ \\
\hline Exogenous NO & 11 & $149.2(265.7)[78]^{\star}$ & $157.1(263.7)[78] \dagger$ \\
\hline Transplantation & 7 & $54.7(44.4)[41]^{\star}$ & $61.0(43.4)[55] \dagger$ \\
\hline Transplantation + infection & 9 & $47.1(16.8)[48]^{\star}$ & 58.7 (14.3) [61] \\
\hline Transplantation + sepsis & 3 & $67.7(9.6)[66]^{\star}$ & $200.7(150.5)[154] \dagger \ddagger$ \\
\hline Transplantation + rejection & 3 & $121.25(92.2)[81]^{\star}$ & $161.7(70.4)[144]+\ddagger$ \\
\hline
\end{tabular}

${ }^{\star} \mathrm{p}<0.05 v$ controls at presentation; $\dagger \mathrm{p}<0.05 v$ control at maximum; $\neq \mathrm{p}<0.05 v$ transplantation alone at maximum; $\delta \mathrm{p}<0.05 v$ infection and shock without sepsis at presentation; I $\mathrm{p}<0.05 v$ infection and shock without sepsis at maximum. Kruskal-Wallis test with Dunn's test for multiple comparisons.

Millipore suction tray. The cadmium lined wells were washed once with $50 \mu \mathrm{l}$ of ammonium chloride borate buffer, and then an equal volume of the Griess reagent was added to each sample and the absorbance read at $550 \mathrm{~nm}$ using potassium nitrate as a standard. Nitrite concentrations were low $(<10 \mu \mathrm{M})$ in the samples relative to nitrate concentrations. Therefore, the combined nitrate plus nitrite concentration $(\mu \mathrm{M})$ was used for data analysis.

The results of the assay procedure were compared to results obtained using high performance liquid chromatography with a copper coated cadmium column as previously published. ${ }^{4}$ The correlation coefficient for the two methods was 0.946 with a slope of 0.923 . Each assay also included controls of pooled normal serum to which $100 \mu \mathrm{M}$ or $200 \mu \mathrm{M}$ sodium nitrate were added. The pooled normal serum alone contained 25.9 (SD 2.1) $\mu \mathrm{M}$ nitrate. Adding $100 \mu \mathrm{M}$ resulted in recovery of 139.5 (4.2) $\mu \mathrm{M}$ and adding $200 \mu \mathrm{M}$ resulted in recovery of $226.3(9.0) \mu \mathrm{M}$.

DATA ANALYSIS AND DESCRIPTION

The degree to which serum nitrite plus nitrate levels represent exogenously administered NO was evaluated in two ways: (1) linear regression analysis was performed using total dosage of nitroprusside plus glyceryl trinitrate $(\mu \mathrm{g} / \mathrm{kg} /$ $\mathrm{min}$ ) as the independent variable and the change in serum nitrite plus nitrate level from baseline as the dependent variable, and (2) the maximum serum nitrite plus nitrate concentrations attained by children receiving exogenous NO were compared to that of the control group.

The degree to which serum nitrite plus nitrate concentrations represented increased endogenous NO production was evaluated by comparing the maximum level of serum nitrite plus nitrate reached by children during the course of (1) infection, (2) sepsis, (3) shock without sepsis, (4) transplantation, (5) transplantation with infection, (6) transplantation with sepsis, or (7) transplantation with rejection, to the maximum level attained by children in the control group. Because the data were not normally distributed, the Kruskal-Wallis test with Dunn's test for multiple comparisons was used and a $p$ value $<0.05$ was considered significant. Distributions of the maximum serum nitrite plus nitrate levels attained by patients in the various study groups are further described in histogram formats.

The value of the serum nitrite and nitrate concentration as an indicator of disease was assessed by comparing the levels at the time of diagnosis among the different groups using the Kruskal-Wallis test with Dunn's test for multiple comparisons. A $p$ value of $<0.05$ was considered significant. For any group with a $p<0.05$ at the time of diagnosis, the sensitivity, specificity, positive predictive value, and negative predictive value of the serum nitrite plus nitrate level as an indicator of the disease state was calculated at the threshold levels of 2 to 5 standard deviations (SD) above the respective mean for the control group.

The degree to which differences in renal or hepatic metabolism affected serum levels of nitrite plus nitrate was evaluated as follows. The effect of renal dysfunction was evaluated in patients who showed a doubling of serum creatinine in at least three consecutive serum samples and who had concomitant measurements of nitrite and nitrate levels. Individual linear regression analyses were performed using serum creatinine as the independent variable and serum levels of nitrite plus nitrate as the dependent variable. Renal dysfunction was also analysed by performing linear regression analysis using first day values of serum creatinine and concomitant serum concentrations of nitrite plus nitrate for each patient. The effect of hepatic dysfunction on serum concentrations of nitrite and nitrate was examined in patients having at least three serum bilirubin measurements with concomitant measurements of nitrite and nitrate and no evidence of haemolysis. For each of these patients, linear correlation analysis was carried out using serum bilirubin levels as the independent variable and serum concentrations of nitrite plus nitrate as the dependent variable.

Examples of individual patients showing daily changes in clinical status and the associated changes in total serum nitrite plus nitrate are given as well.

\section{Results}

One hundred and thirty seven consecutive critically ill children were evaluated. Thirteen children met criteria for more than one of the study groups at the time the maximum serum nitrite and nitrate concentration was attained and were therefore excluded from analysis. Therefore 124 children meeting criteria for only one group at the time of diagnosis and at the point of maximum serum nitrite plus nitrate concentration were included in the analysis. The control group reflected the diversity of the patient population typically seen in our intensive care unit. Postoperative diagnoses included intracranial and spinal surgery ( $n=$ 13), head and neck surgery $(n=4)$, and cardiac surgery $(n=13)$. Five children with trauma but no shock were in the control group. Medical diagnoses included Guillain-Barré syndrome, cystic fibrosis awaiting lung transplantation, imipramine ingestion, airway obstruction, sickle cell disease with chest syndrome, bronchopulmonary dysplasia, diabetic ketoacidosis, 


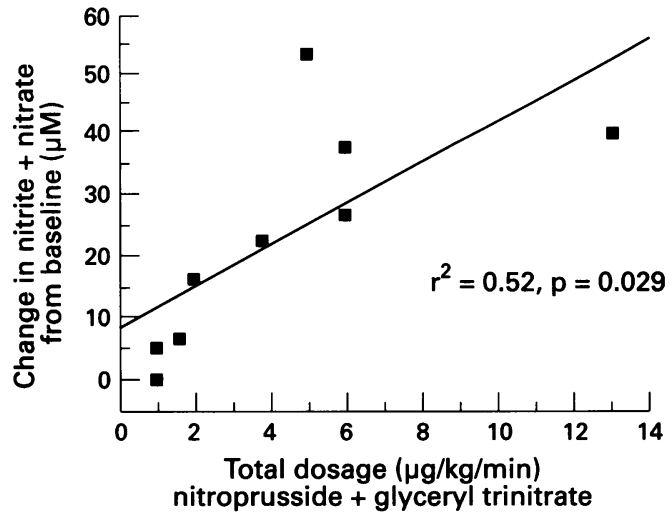

Figure 1 Linear correlation analysis between changes in serum nitrite plus nitrate concentrations and dose of glyceryl trinitrate plus nitroprusside given to nine children.

hypothermia, bronchiolitis, encephalopathy, status epilepticus, hypertensive crisis, status asthmaticus, and near miss sudden infant death syndrome. No medical diagnosis in the control group contained more than two patients during the study period. In these patients the maximum serum nitrite plus nitrate concentration ranged from 4 to $61 \mu \mathrm{M}$ (median 18).

Children receiving exogenous NO donors or inhaled NO reached a higher maximum serum nitrite plus nitrate concentration than the control group $(\mathrm{p}<0.05)$ (table 2$)$. Nine children who received glyceryl trinitrate and/or nitroprusside were evaluated to determine whether serum nitrite plus nitrate concentrations were representative of the amount of NO donor received. Figure 1 shows that changes in serum nitrite plus nitrate concentrations correlated with the total doses of glyceryl trinitrate and/or nitroprusside received $\left(r^{2}=0.52, \mathrm{p}=0.02\right)$.

At the time of diagnosis children with sepsis or transplantation had higher serum nitrite
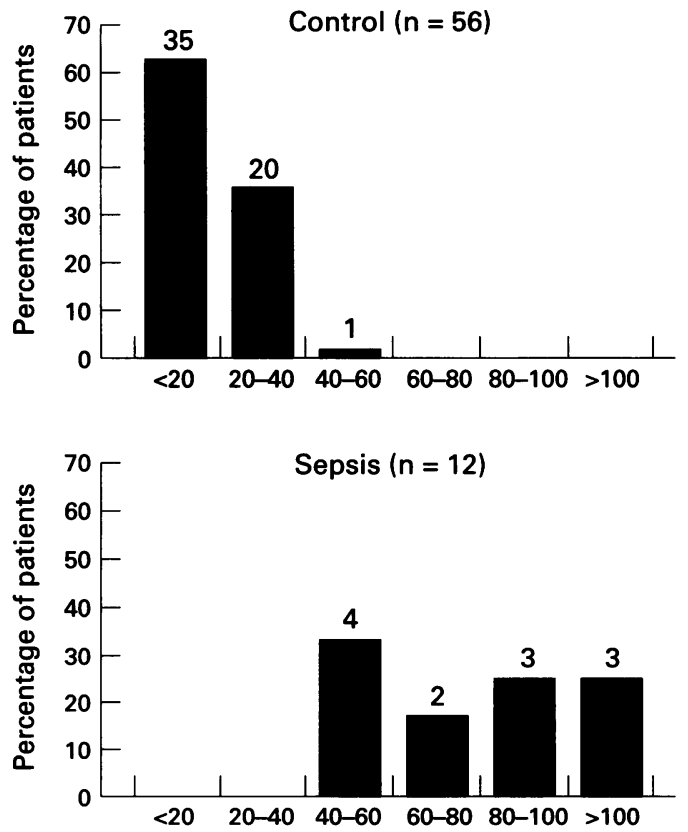

Ranges of maximum serum nitrate plus nitrate $(\mu \mathrm{M})$

plus nitrate concentrations than controls $(p<0.05)$ (table 2). Values in children with infection, or shock not associated with sepsis, were not different from controls at the time of diagnosis. Over the course of the illness children with infection, sepsis, shock without sepsis, or transplantation reached a higher maximum serum nitrite plus nitrate concentration than control patients $(p<0.05)$ (table 2$)$. Children with shock not associated with sepsis had multiple aetiologies for shock including haemorrhage $(n=3)$, congestive heart failure $(n=4)$, and cardiopulmonary arrest $(n=1)$. At the time of diagnosis, these children had a lower serum nitrite and nitrate level than children with sepsis $(p<0.05)$. Also, the maximum level attained by these children was lower than that attained by children with sepsis $(\mathrm{p}<0.05)$ (table 2).

After organ transplantation, patients with concomitant rejection or sepsis showed no difference in serum nitrite plus nitrate concentrations at the time of diagnosis than those without concomitant sepsis or rejection. However, the maximum concentration attained by children with sepsis or rejection after organ transplantation was higher than that attained by patients without sepsis or rejection after organ transplantation $(\mathrm{p}<0.05)$ (table 2$)$.

Figure 2 shows that $36 \%$ of control patients attained a maximum serum nitrite plus nitrate concentration greater than $20 \mu \mathrm{M}$, and only $2 \%$ attained a maximum concentration greater than $40 \mu \mathrm{M}$. Eighty eight per cent of patients with infection attained a maximum concentration greater than $20 \mu \mathrm{M}$, and $52 \%$ attained a concentration greater than $40 \mu \mathrm{M}$. All sepsis syndrome patients attained a maximum concentration greater than $40 \mu \mathrm{M}$, and $50 \%$ attained a concentration greater than $80 \mu \mathrm{M}$. No patient in the control or infection group
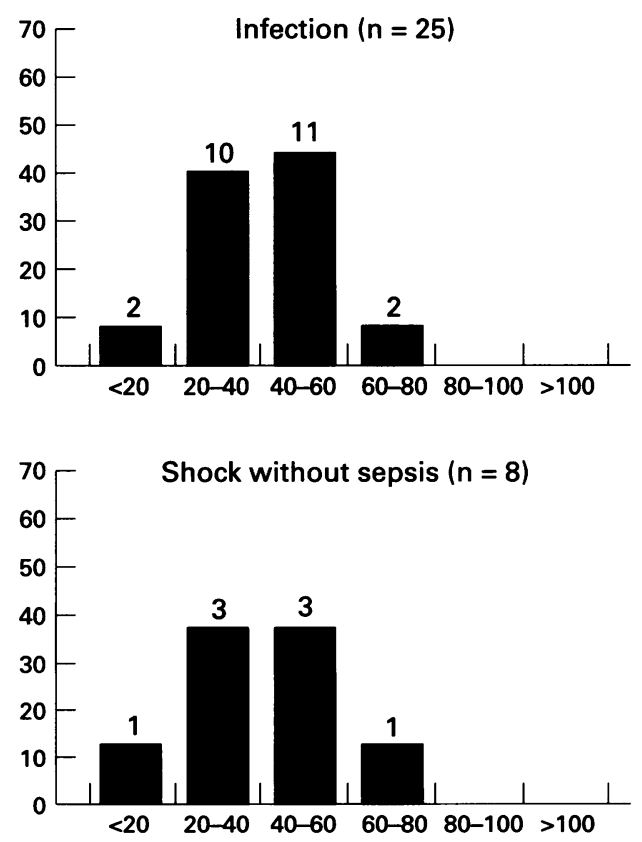

Figure 2 Histogram analyses for the control, infection, sepsis, and shock without sepsis groups depicted as the percent of patients having a maximum serum nitrite plus nitrate concentration within the specified ranges. See text for definitions of groups. Numbers above bars indicate number of patients in each range. 

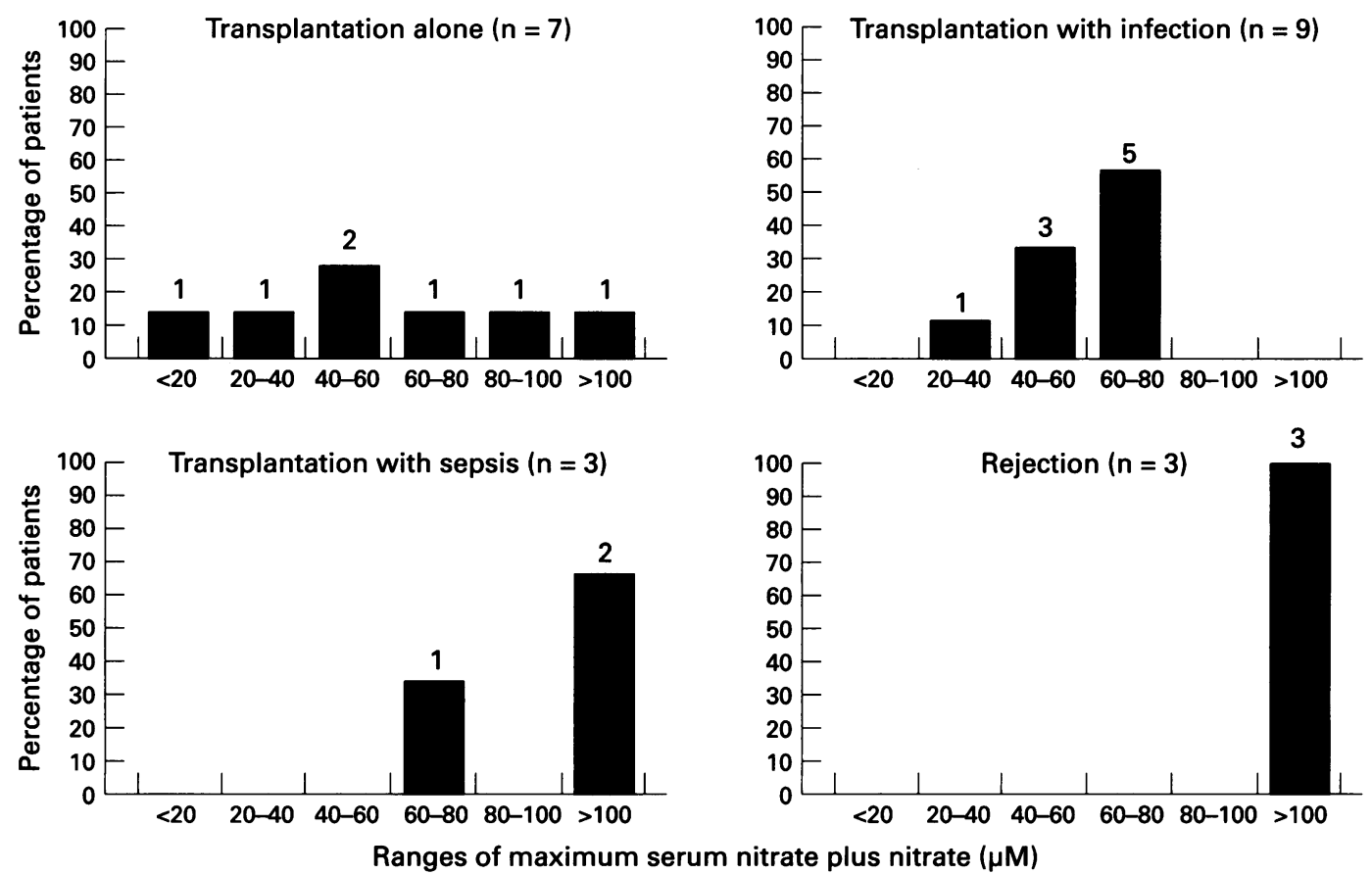

Figure 3 Histogram analyses for the transplantation, transplantation with infection, transplantation with sepsis, and rejection groups depicted as the percent of patients having a maximum serum nitrite plus nitrate concentration within the specified ranges. See text for definitions of groups. Numbers above bars indicate number of patients in each range.

( $\mathrm{n}=81$ ) attained a maximum concentration greater than $80 \mu \mathrm{M}$. Children with shock not associated with sepsis did not attain a maximum concentration greater than $80 \mu \mathrm{M}$. The maximum serum nitrite plus nitrate concentrations attained in children with transplantation were widely distributed (fig 3). However, all three patients with acute rejection attained a maximum concentration greater than $100 \mu \mathrm{M}$.

Among non-transplant patients, only patients with sepsis syndrome had higher serum nitrite plus nitrate than controls at the time of diagnosis. Based on this analysis the sensitivity, specificity, positive predictive value, and negative predictive value of serum nitrite plus nitrate concentrations at the time of diagnosis of sepsis was evaluated at 2, 3, 4, and 5 SD above the respective mean for the control group (table 3). All patients from the control, infection, sepsis, and sepsis without shock groups were included in this evaluation. No patients with transplantation were included. Notably, a serum level of $35.8 \mu \mathrm{M}$ (2 SD above the control mean) had a negative predictive value of $99 \%$ for sepsis with a sensitivity of $92 \%$ and a specificity of $86 \%$. In contrast, a serum concentration of $63.6 \mu \mathrm{M}$ (5 SD from the control mean) had a positive predictive value of $100 \%$ for sepsis with a sensitivity of $50 \%$ and a specificity of $100 \%$.

Eighteen children showed a doubling of serum creatinine with at least three concomi-

Table 3 The sensitivity, specificity, positive predictive value, and negative predictive value of serum nitrite plus nitrate levels at the time of diagnosis in non-transplant patients with sepsis. See text for details of analysis; values are per cent

\begin{tabular}{llcll}
\hline $\begin{array}{l}\text { Value (SD above } \\
\text { control mean) }\end{array}$ & Sensitivity & Specificity & $\begin{array}{l}\text { Positive } \\
\text { predictive value }\end{array}$ & $\begin{array}{l}\text { Negative } \\
\text { predictive value }\end{array}$ \\
\hline $35.8 \mu \mathrm{M}(2)$ & 92 & 86 & 48 & 99 \\
$45.1 \mu \mathrm{M}(3)$ & 67 & 93 & 57 & 95 \\
$54.3 \mu \mathrm{M}(4)$ & 67 & 95 & 67 & 95 \\
$63.6 \mu \mathrm{M}(5)$ & 50 & 100 & 100 & 94 \\
\hline
\end{tabular}

tant serum creatinine measurements. Two (11\%) of these patients had a significant correlation between serum creatinine and serum nitrite plus nitrate concentrations on these days $\left(p<0.05 ; r^{2}=0.63\right.$ and 0.57 , respectively). In 86 patients, first day serum creatinine values did not correlate with concomitant serum nitrite plus nitrate concentrations $\left(p=0.10 ; r^{2}=0.032\right)$. Eleven patients had at least three serum bilirubin estimations with concomitant serum nitrite plus nitrate concentrations available for analysis. One (9\%) of these patients had a significant correlation between these values $\left(p<0.05, r^{2}=0.79\right)$.

Figure 4A shows a patient with increasing serum nitrite plus nitrate concentrations that were associated with therapeutic inhalation of NO. Discontinuation of inhaled NO was associated with a decrease in the concentrations towards baseline. Figure 4B shows a child with septic shock that was associated with increasing serum nitrite plus nitrate concentrations. Figure 4C shows a child with acute allograft rejection who showed an increase in serum nitrite plus nitrate concentrations during the episode of rejection. The values decreased after immunosuppressive treatment with OKT-3. Figure 4D represents a patient who developed acute renal failure, sepsis, and rejection at different timepoints. In this patient, serum nitrite plus nitrate concentrations did not change with changes in renal function as measured by serum creatinine. However, changes in serum nitrite plus nitrate did occur during episodes of septic shock and acute rejection.

\section{Discussion}

To our knowledge this survey is the first to establish a range of serum nitrite plus nitrate concentrations in the general critically ill 
paediatric population. As hypothesised, patients receiving exogenous sources of $\mathrm{NO}$ (glyceryl trinitrate, nitroprusside, or inhaled NO) had higher levels than control patients. The observed correlation between dosage changes in NO donor medications and serum nitrite plus nitrate concentrations suggests that this test may be a useful indirect measure of NO in children.

As hypothesised, serum nitrite plus nitrate concentrations were increased in children with sepsis or transplantation at the time of diagnosis. Interestingly, significantly increased values were not seen in children with infection, or shock without sepsis, two groups expected to have increased endogenous NO production relative to control patients at the time of diagnosis. However, over the course of illness both groups reached a maximum level greater than that of the control population. Similar results occurred in the transplant population. At the time of diagnosis, children with transplantation having concomitant sepsis or rejection did not have significantly higher concentrations than transplant patients without sepsis or rejection. However, over time the maximum concentration attained was higher when sepsis or rejection was present concomitantly. One possible explanation for these findings is that NO production may increase at the onset of these conditions, but increased serum nitrite plus nitrate levels are not detectable for some period of time.
The lack of a strong association between evolving renal or hepatic dysfunction and serum nitrite plus nitrate concentrations supports the notion that increased concentrations during infection, sepsis, shock without sepsis, transplantation, or rejection are due, at least in part, to increased endogenous NO production rather than to decreased metabolism or elimination of stable end products. This assertion must be considered carefully because differences in metabolism could be important in individual patients.

Endogenous NO production occurs through the enzyme nitric oxide synthase (NOS), of which there are three isoforms: endothelial NOS, neuronal NOS, and inducible NOS (iNOS). ${ }^{15}$ iNOS is expressed after exposure to endotoxin or cytokines, or both, and is thought to be responsible for prolonged and high output of NO seen during proinflammatory conditions such as the sepsis syndrome. ${ }^{16}$ Animal and human studies have shown that increased serum nitrite and nitrate concentrations reflect increased endogenous production of NO during the sepsis syndrome. ${ }^{34}$ Interestingly, iNOS regulation can manifest marked species variability and specificity, implying that iNOS regulation in humans may not be governed by the same proinflammatory mediators that lead to iNOS expression in rodents and other mammalian species. ${ }^{17}$ Our present data indicate that increased endogenous production of NO occurs not only during the sep-
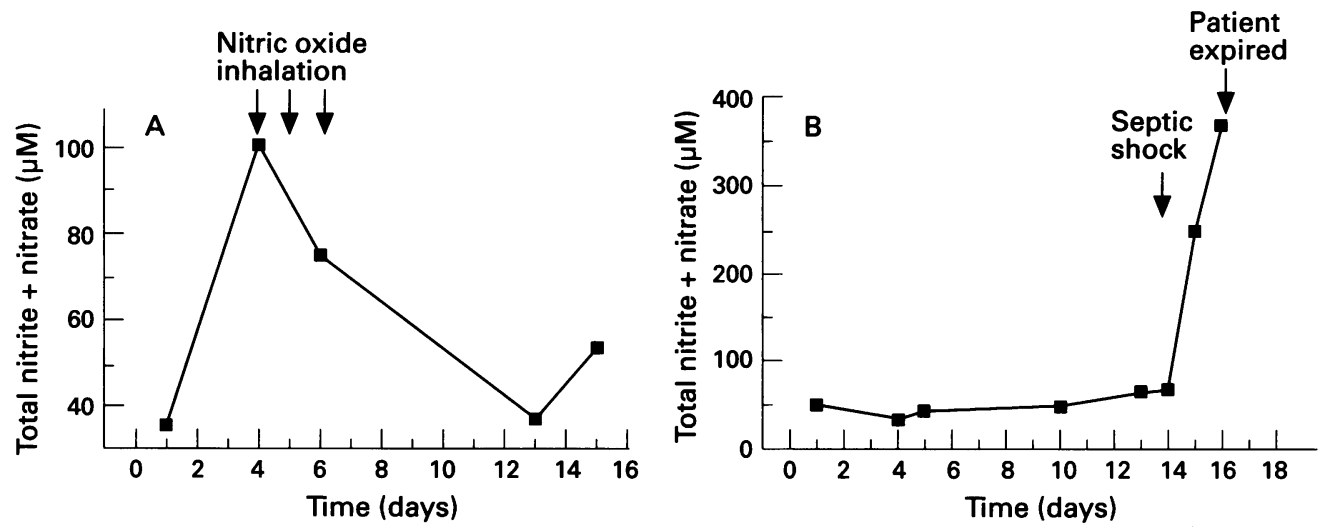

Figure 4 (A) Changes in serum nitrite plus nitrate concentrations in a patient during and after treatment with inhaled NO. (B) Changes in serum nitrite plus nitrate concentrations in a patient who developed septic shock. (C) Changes in serum nitrite plus nitrate concentrations in a child who was treated for acute allograft rejection following heart transplantation. (D) Changes in serum nitrite plus nitrate concentrations in a child who developed septic shock, acute renal failure, and acute organ rejection at different timepoints. 
sis syndrome, but also in other clinical conditions associated with immune activation such as infection, transplantation, and rejection. The NOS isoform responsible for this increased endogenous NO remains to be definitively established.

Patients with infection tended to have lower serum levels of nitrite and nitrate than patients with the sepsis syndrome. We speculate that some patients with a focus of infection who go on to develop the sepsis syndrome may have a more extensive elaboration of proinflammatory mediators and consequently produce greater amounts of NO (possibly through iNOS) than patients with infection who do not develop the sepsis syndrome. If this speculation is correct, then it would lend further support for the role of $\mathrm{NO}$ as an important effector molecule in the manifestations of the sepsis syndrome.

The observation that patients with either transplantation or rejection had increased serum nitrite plus nitrate concentrations provides a relevant clinical correlate to rodent models of transplantation. ${ }^{18}{ }^{19}$ In these models serum nitrite and nitrate concentrations increase in proportion to the degree of rejection, suggesting that greater immune stimulation (as occurs during acute allograft rejection) leads to greater endogenous NO production. A similar phenomenon may have occurred in our patients, as evidenced by the maximum serum nitrite plus nitrate values greater than $100 \mu \mathrm{M}$ in all three patients with acute rejection but in only one of seven patients with transplantation in the absence of acute rejection.

We also observed higher maximum serum nitrite plus nitrate concentrations in children who developed shock as a result of chronic heart failure, haemorrhage, or cardiorespiratory arrest. These conditions are not commonly linked to inflammation, and are not generally thought to be associated with increased iNOS expression. Rather, the endothelial NOS pathways have been hypothesised as the source of increased NO production. Interestingly, patients with sepsis had higher serum nitrite plus nitrate concentrations than children with shock not associated with sepsis. One possible explanation for this observation is that immune activation increases NO production in children with sepsis in addition to any increase in NO which might be associated with hypotension per se. Future studies will be needed to address this possibility.

The clinical value of serum nitrite and nitrate concentrations as a tool for diagnosis or therapeutic monitoring of sepsis or allograft rejection requires further study. The observation that serum nitrite plus nitrate concentrations above $80 \mu \mathrm{M}$ were attained only by children with sepsis, transplantation, or organ rejection suggests that a threshold serum nitrite plus nitrate concentration may be used as an indicator of immune stimulation and increased endogenous NO production. The observation that individual patients had increased levels above $80 \mu \mathrm{M}$ when rejection or sepsis occurred, and returned to lower levels when sepsis or rejection resolved, suggests that absolute changes in serum nitrite and nitrate concentrations could be indicative of these disease states. In some children, it may be possible to follow concentrations as a measure of therapeutic efficacy. However, the observation that some children with sepsis never attain concentrations greater than those in control patients suggests that this test alone will not be diagnostic in all children with sepsis. Also the observation that the level at presentation with sepsis can be lower than the maximum value attained over time suggests that the diagnostic value of the test will depend on the time when the assay is performed. Similarly, the overlap between the maximum serum nitrite plus nitrate concentrations attained by transplantation patients, with and without acute allograft rejection, suggests that this test will be unable to differentiate all acute rejection events. At present no laboratory test other than biopsy has been shown to possess this degree of discrimination. Future investigation is warranted to determine whether serum nitrite and nitrate concentrations can add any further predictive power to tests currently used to evaluate children at risk for sepsis or rejection.

Limitations of this study should be considered. Definitions of sepsis and rejection may be subject to interpretation. For example, we did not subcategorise children with the systemic inflammatory response syndrome. ${ }^{20}$ Some patients in the infection group may have been classified as having the systemic inflammatory response syndrome, rather than infection, by other investigators. Also, we did not require biopsy verification of acute rejection. Some patients with transplantation may have had varying degrees of acute rejection, but according to our definitions were placed in the transplantation group rather than the rejection group. Third, increased levels in patients with acute rejection may have occurred secondary to immunotherapy rather than the rejection process alone.

In summary, we suggest that endogenous NO production can be evaluated in critically ill children using a method for measurement of nitrite and nitrate which allows for rapid processing of samples in the clinical laboratory setting. It is important to note that measurement of nitrite levels alone would not have been sufficient to carry out this study; it was necessary to develop a technique that allows for concomitant measurement of nitrate as well. The pending development of pharmacological strategies directed towards the selective inhibition of iNOS underlines the relevance of these data. We conclude that endogenous NO production, as measured by serum nitrite and nitrate levels, is increased in clinical conditions characterised by immune stimulation: infection, sepsis, transplantation, and rejection, and in a clinical condition not commonly associated with immune activation-shock without sepsis. Our data provide a foundation for future studies concerning the role and sources of endogenous NO production in pediatric critical illness. The value of serum nitrite and nitrate concentrations as a useful diagnostic tool or therapeutic monitor in the clinical evaluation of children at risk for developing the 
sepsis syndrome or acute allograft rejection remains to be established.

This work was supported in part by a grant from the National Institute of Health (RO1GM41734-04A1) to SK.

1 Wagner DA, Young VR, Tannenbaum SR. Mammalian nitrate biosynthesis: incorporation of ${ }^{15} \mathrm{NH}_{3}$ into nitrate is enhanced by endotoxin treatment. Proc Natl Acad Sci USA 1983;80:4518-21.

2 Nathan C. Nitric oxide as a secretory product of mammalian cells. FASEB $\mathcal{F}$ 1992;6:3051-64.

$3 \mathrm{Shi} \mathrm{Y}, \mathrm{Li} \mathrm{HQ}$, Shen CK, et al. Association between protecShi Y, Li HQ, Shen CK, et al. Association between protec-
tive efficacy of antibodies to tumor necrosis factor and suppression of nitric oxide production in neonatal rats with fatal infection. Pediatr Res 1993;34:345-8.

4 Ochoa JB, Udekwu AO, Billiar TR, et al. Nitrogen oxide levels in patients after trauma and during sepsis. Ann Surg 1991;214:621-6.

$5 \mathrm{Shi} \mathrm{Y}, \mathrm{Li} \mathrm{HQ}$, Shen CK, et al. Plasma nitric oxide levels in newborn infants with sepsis. 7 Pediatr 1993;123:435-8.

6 Jacob TD, Ochoa JB, Udekwu AO, et al. Nitric oxide production is inhibited in trauma patients. F Trauma 1993 35:590-6.

7 Wong HR, Carcillo JA, Burckart G, Shah N, Janosky JE. Increased serum nitrite and nitrate levels in children with the sepsis syndrome. Crit Care Med 1995;23:835-42.

8 Wong HR, Finder JD, Wasserloos K, Pitt BR. Expression of inducible nitric oxide synthase in cultured rat pulmonary artery smooth muscle cells is inhibited by the heat shock response. Am f Physiol 1995;269:L843-8.

9 Geller DA, Nussler AK, DiSilvio M, et al. Cytokines, endotoxin, and glucocorticoids regulate the expression of inducible nitric oxide synthase in hepatocytes. Proc Natl Acad Sci USA 1993,90:522-6.
10 Langrehr JM, Hoffman, RA, Lancaster JR Jr., Simmons, RL. Nitric oxide-a new endogenous immunomodulator. Transplantation 1993;55:1205-12.

11 Yokokawa $\mathrm{K}$, Mankus $\mathrm{R}$, Saklayer $\mathrm{M}$, et al. Increased nitric oxide production in patients with hypotension during hemodialysis. Ann Intern Med 1995;123:35-7.

12 Habib F, Duthra D, Gossman D, Oakley CM, Cleland JG Enhanced basal nitric oxide production in heart failure: Enhanced basal nitric oxide production in heart failure: another failed counter-regu

13 Bone RC, Fisher CJ, Clemmer TP, Slotman GJ, Metz CA, Balk RA. Sepsis syndrome: a valid clinical entity. Crit Care Med 1989;17:389-93.

14 Green LC, Wagner DA, Glogowski J, et al. Analysis of nitrate, nitrite, and ${ }^{15} \mathrm{~N}$ nitrate in biological fluids. Anal Biochem 1982;126:131-8.

15 Nathan C, Qiao-wen X. Nitric oxide synthases: roles, tolls, and controls. Cell 1994;78:915-8.

16 Rees DD, Cellek S, Palmer RM, Moncada S. Dexamethasone prevents the induction by endotoxin of a nitric oxide synthase and the associated effects on vascular tone: an insight into endotoxin shock Biochem Biophys Res Commun 1990;173:541-7.

17 Felley-Bosco E. Species specificity at the molecular level: The case of nitric oxide synthesis. Arch Toxicol 1995; suppl 17:357-66.

18 Langrehr JM, Murase N, Markus PM, et al. Nitric oxide production in host-versus-graft and graft-versus-host reaction in the rat. $\mathcal{f}$ Clin Invest 1992;90:679-8

19 Langrehr JM, Müller AR, Lee TK, Schraut WH, Simmons $\mathrm{RL}$, Hoffman RA. Serum $\mathrm{NO}_{2}^{-} / \mathrm{NO}_{3}^{-}$from oxidative L-arginine metabolism: a possible marker for small bowel allograft rejection [abstr]. Transplant Proc 1992;24:1148.

20 Bone RC, Balk RA, Cerra FB, et al. Definitions for sepsis and organ failure and guidelines for the use of innovative therapies in sepsis. The ACCP/SCCM Consensus Conference Committee. American College of Chest Physicians/ Society of Critical Care Medicine. Chest 1992;101:1644 55. 